\title{
Management of Medication-Related Cardiometabolic Risk in Patients with Severe Mental Illness
}

\author{
Donna J. Lang • Alasdair M. Barr • Ric M. Procyshyn
}

Published online: 14 June 2013

(C) The Author(s) 2013. This article is published with open access at Springerlink.com

\begin{abstract}
Severe psychotic disorders, which on their own may be a risk factor for metabolic disorder and cardiovascular illness, are clinically compounded by the significant adverse side effects of antipsychotic medications. The majority of patients with severe psychotic disorders (i.e., schizophrenia, bipolar disorder, mania, and depression) must take antipsychotic medications to treat their psychoses and, subsequently, will require efficacious interventions to manage the metabolic consequences of pharmacologic treatment to mitigate excessive mortality associated with cardiovascular illness. We have reviewed the metabolic consequences of antipsychotic treatment and discussed pilot findings from a new nonpharmacologic intervention study looking at the clinical benefits of regular exercise as a management tool for the cardiometabolic risk factors in a cohort with severe mental illness.
\end{abstract}

Keywords Mental illness · Cardiometabolic disorder . Antipsychotics $\cdot$ Exercise

D. J. Lang $(\bowtie)$

Department of Radiology, University of British Columbia, Centre for Complex Disorders, British Columbia Mental Health and Addictions Research Institute, CFRI Bldg, Rm. 3A-124, 938 West 28th Ave, BC Children's Hospital Site,

Vancouver, BC, Canada V5Z 4H4

e-mail: dlang@mail.ubc.ca

\section{A. M. Barr}

Department of Pharmacology, British Columbia Mental Health and Addictions Research Institute, Vancouver, BC, Canada V5Z 4H4

R. M. Procyshyn

Department of Psychiatry, British Columbia Mental Health and Addictions Research Institute, Vancouver, BC, Canada V5Z 4H4

\section{Introduction}

Metabolic and Cardiovascular Disease in Severe Mental Illness

Of all the mental disorders, those that fall under the umbrella of psychotic disorders are certainly the most disabling and most difficult to manage. Severe psychiatric deficits, including hallucinations, delusions, cognitive difficulties, and poor social and occupational functioning, plus a debilitating course of lifelong cognitive impairments are characteristic of patients diagnosed with schizophrenia, severe bipolar disorder, and chronic clinical depression [1]. These disorders are also characterized by significantly decreased life expectancy with the chief factors of this excess risk of death arising from cigarette smoking, obesity, metabolic disorders associated with diabetes, hypertension, and stroke [2,3]. A recent survey of over 1000 subjects with psychotic disorders conducted in Australia noted that over $75 \%$ of all patients were overweight or obese, $20 \%$ suffered from diabetes and hypertension, and over $50 \%$ of patients met criteria for metabolic syndrome, defined by a combination of central obesity plus 2 or more of the following risk factors: elevated HDL C levels, increased blood pressure, increased blood glucose levels [4]. Other recent studies done in South America and in Asia have reported that prior to treatment, young patients may already be at a metabolic disadvantage, with already higher than average rates of obesity (21\%), hypertension ( $29 \%$ ), and smoking (26\%) and significantly increased rates of diabetes $(8 \%$ vs $1 \%$ in agematched controls) $[5,6]$. These physical health deficits are considerably exacerbated by the antipsychotic and antidepressant medications required to treat their mental illnesses.

For many patients, reduction of dosing and/or tapering off psychotropic medications is not clinically recommended, given the seriousness of these mental disorders and the high risk of relapse and significant self-harm and harm to others. The long-term overall result is an approximate 20-year 
reduction of lifespan from early death from cardiovascular and metabolic disease [7]. Mortality rates are likely to be similar in Canada and the US. Alternate non-pharmacologic approaches and interventions for concurrent metabolic disorders in severe mental illness are required to modify the cardiometabolic sequelae of treatment to alleviate the burden of mortality in this population.

Medication Treatment: Cardiovascular and Metabolic Effects

In the previous decade, increased rates of abdominal obesity, dyslipidemia, hyperglycemia, and hypertension have been observed in patients with psychotic disorders, all of which are key risk factors for cardiometabolic disorders, such as diabetes and cardiovascular disease [8,9]. Patients presenting with severe psychosis and schizophrenia spectrum disorders are widely prescribed antipsychotic medications to manage the symptoms of illness. There are an estimated 235,000 people with schizophrenia in Canada, with an economic impact at over $\$ 6.85$ billion annually [10]. The use of antipsychotic drugs has increased dramatically in the past decade, with the introduction of newer compounds with different sideeffect profiles, additional approved indications as well as greater off-label use. For example, members of our group have previously reported that antipsychotics were being used off-label for a wide range of disorders, including bipolar, depression, anxiety, and personality disorders in a large Canadian cohort [11]. We and others have reported that antipsychotics are also being prescribed increasingly to nontraditional populations such as children and adolescents.

The first generation antipsychotics (FGAs), or "typical", antipsychotic drugs were developed in the 1950s, and revolutionized psychiatry. However, many patients remained resistant or refractory to their clinical effects, and extended use commonly resulted in severe neurological motor side-effects, and subsequently fell out of favor $[12,13]$. The second generation antipsychotics (SGAs) (also known as the "atypicals"), developed in the 1990 and 2000s, represent compounds with greater efficacy for refractory psychosis. While the SGAs display much lower propensity to induce motor side-effects, recent research has indicated that these drugs are commonly associated with different but equally severe side-effects. All antipsychotic medications are associated with higher rates of sedation, sexual dysfunction, postural hypotension, cardiac arrhythmia, and sudden cardiac death [13, 14]. Preclinical animal studies have clearly shown that exposure to SGAs have direct adverse effects on the cardiovascular system [15]. At this time there is overwhelming evidence indicating that use of the SGAs can result in severe metabolic disturbances that requires much better clinical management than currently provided in order to prevent unnecessary mortality $[8,16]$. These effects are so severe that the American Diabetes Association, the American Psychiatric Association, the American Association of Clinical Endocrinologists and the North American Association for the Study of Obesity co-sponsored a Joint Consensus Statement, providing guidelines for assessment of metabolic risk for all patients being treated with atypical antipsychotics [17].

Weight gain is the most common adverse effect among the SGAs and some of the FGA. In a review of the literature, Allison et al. [18] noted the following mean increases in weight at 10 weeks of treatment with the SGAs: clozapine $4.45 \mathrm{~kg}$, olanzapine $4.15 \mathrm{~kg}$, chlorpromazine $2.58 \mathrm{~kg}$, risperidone $2.10 \mathrm{~kg}$, haloperidol (a FGA) $1.08 \mathrm{~kg}$, and ziprasidone $0.04 \mathrm{~kg}$. In another systematic review, increases in weight of $10 \%$ or more associated with clozapine, olanzapine, and risperidone were: $6 \%$ at 8 weeks, $15 \%$ at 10 weeks, and $27 \%-60 \%$ at 3-12 months [19]. These results have been corroborated in several meta-analyses [20]. Our own observations in a cohort of drug-naïve first episode patients showed an average weight gain of $1.8 \mathrm{~kg}$ in just 4 weeks of low dose risperidone or quetiapine exposure (unpublished data). Concomitant dislipidemia has also been noted in those treated with SGAs [21-23]. The SGA quetiapine is also often associated with increased triglyceride levels [24].

Alarmingly, among patients with metabolic syndrome, the relative risk for type II diabetes and coronary heart disease (CHD) is 1.5-5 times that of the general population [25]. Results from the Clinical Antipsychotic Trial of Intervention Effectiveness (CATIE) study (a large multi-center trial sponsored by NIMH) controlled for factors including age, race and gender and observed that $42.7 \%$ of patients treated with atypicals had metabolic dysregulation. When controlling for Body Mass Index (BMI), CATIE men were $85 \%$ more likely, and CATIE women $137 \%$ more, to have metabolic syndrome than their control counterparts [26]. The greater risk for metabolic dysregulation associated with SGAs may be independent of effects on body weight [27, 28]. It is therefore very likely that the SGAs exert effects on glucose tolerance and insulin sensitivity that are independent of (and not secondary to) weight gain.

Further evidence in support of this hypothesis comes from reports that have detailed new-onset diabetes in the absence of obesity or substantial weight gain in atypical drug-treated patients [29]. Additionally, acute effects of SGAs in normal subjects included glucose intolerance in the absence of major weight gain [30]. Over $25 \%$ of the diabetic keto-acidosis and $15 \%$ of new-onset hyperglycemia cases were in atypical-treated patients who did not experience weight gain or who had lost weight [29].

Management Strategies and Nonpharmacological Interventions: Clinical Monitoring and Exercise

Despite incontrovertible evidence of increased cardiometabolic risk in individuals with mental illness taking antipsychotic 
medication, metabolic screening practices are often incomplete or inconsistent [31]. Unfortunately, patients with schizophrenia are often at risk of receiving less optimal treatment for cardiovascular disease compared with other populations [32]. A recent review by Mitchell and colleagues found that across 39 internationally published studies, rates of routine baseline metabolic screening was generally low, and above $50 \%$ only for blood pressure and triglycerides (59.9\%). Cholesterol was measured in $41.5 \%$, glucose in $44.3 \%$, and weight in $47.9 \%$ of patients. Lipids and glycosylated haemoglobin (HbAlc) were monitored in less than $25 \%$ [31]. These data indicated that in routine clinical practice, metabolic monitoring is remarkably low in people prescribed antipsychotic medications, despite the introduction of new monitoring guidelines for this population [33]. The following measurements were recommended in the development of new guidelines (in order of frequency): fasting glucose, body mass index, fasting triglycerides, fasting cholesterol, waist, high-density lipoprotein/low-density lipoprotein, blood pressure, and symptoms of diabetes [33]. In an ongoing study that we are conducting in a cohort of treatment-resistant chronic psychosis patients, we have observed that an additional measure of arterial stiffness based on aortic pulse wave velocity offers a sensitive and effective tool for evaluating cardiovascular disease risk in this population, and may be a more rapid way of accurately assessing cardiovascular disease risk (Phillips et al. submitted) [34].

In terms of additional interventions, most clinical guidelines recommended providing advice on physical activity, diet, psycho-education of the patient (and family), treatment of lipid abnormalities, treatment of diabetes, referral for advice and treatment, and smoking cessation advice. While commendable, the provision of advice alone does not ensure that patients most in need of intervention actually receive any appropriate intervention. Moreover, due to the physical and psychological limitations of this population, physical activity, or exercise interventions, which are likely to confer considerable health benefits with respect to moderating the metabolic side-effects of medications, requires both a tailored exercise prescription and ongoing clinical monitoring. Exercise barriers for those with severe mental disorders generally include lack of motivation, poor concentration, sedative effects of medications, insufficient financial resources, and lack of access to appropriate exercise programs [35].

It has been suggested that the beneficial effects of exercise may be greatly under-appreciated and that the positive effects of controlled exercise include not only improved metabolic responses, but may also confer neuro-protection, increased quality of life, and reduction in the severity of psychopathological symptoms [36]. With respect to the adverse metabolic effects of antipsychotic treatment, physical exercise can prevent or lessen cardiovascular disease risk factors including elevated blood pressure, insulin resistance, glucose intolerance, elevated blood triglycerides, low high-density lipoprotein cholesterol levels, and obesity, all of which are high in prevalence in psychosis patients [37].

It is apparent that severe mental illnesses are diseases of the brain, resulting in adverse clinical sequelae, such as psychosis and cognitive dysfunction. Exercise may provide direct benefit for these aspects of the illness as well. In this regard, a recent review has highlighted the value of regular exercise programs in schizophrenia patients with respect to ameliorating the severity of psychosis [38*0]. Clearly, for those with severe mental illness, appropriate exercise offers both physical and psychiatric benefits, and is thought to exert a salutary effect on interacting networks mediating metabolism, immuno-inflammatory function, and cellular respiration for both body and brain [39]. The therapeutic benefits of regular exercise in those with major depression is well known [40], and is also believed to alleviate symptom severity in schizophrenia patients [41]. Currently, there is a paucity of research testing its effects on people seeking help for mental health issues [42]. Moreover, it is generally observed that compliance with prescribed exercise programs is low [42]. It is likely that expecting people to exercise at levels recommended by standard population guidelines may be unrealistic given their struggle with mental health issues, or that self-directed exercise is possible.

In an ongoing investigation our group is studying the effects of a 12-week regular exercise intervention on physical and mental health in chronic treatment-resistant psychosis patients. Full cardiovascular fitness evaluations have so far been conducted in a cohort of 17 chronic psychosis patients (mean age 31.9 yrs) treated with high dose SGAs (Invited Poster Symposium - International Congress of Schizophrenia Research 2013, Orlando, Florida) [43•]. Individualized exercise programs were tailored to patients based on their baseline cardiorespiratory fitness and motor abilities. For this study, fitness progress was closely monitored on a weekly basis over the 12-week course of intervention. Full cardiorespiratory fitness was re-assessed at 6 weeks and 12 weeks. It is well known that low cardiovascular or cardiorespiratory fitness, as assessed by $\mathrm{VO}_{2}$ Max, is a key factor for coronary heart disease. The mean cardiovascular capacity $\left(\mathrm{VO}_{2} \mathrm{Max}\right.$ $\mathrm{ml} / \mathrm{kg} / \mathrm{min}$ ) in this cohort at baseline was $19.8 \mathrm{ml} / \mathrm{kg} / \mathrm{min}$, far below the expected healthy norms for this age cohort [44], and was congruent with patients suffering from mild to moderate heart failure [45]. The observation of lower than predicted $\mathrm{VO}_{2}$ Max capacity has been observed in less severely ill schizophrenia patients as well $[46,47]$. Despite the presence of SGAs, we, and others have measured improvements in $\mathrm{VO}_{2}$ Max and blood pressure after just 6 to 12 weeks of regular exercise at moderate exertion levels ( $55 \%-70 \%$ of maximum heart rate) $[43 \cdot 46]$. Our own data have shown that after only 12 weeks of regular exercise, patients were able to reduce their 
average body mass index (BMI) from a mean baseline BMI of 29.9 to a mean BMI of 27.112 weeks later [43•]. For this cohort of severely ill patients, improvements in anxiety, depression, and psychotic symptom severity were also observed after completion of 12 weeks of regular exercise [43•].

These preliminary observations strongly suggest that even for the most symptomatically severe and treatment refractory patients, adjunct regular exercise offers significant benefits with respect to lessening metabolic effects of antipsychotic treatment. Moreover, the improvements in mood deficits and cognitive deficits conferred by regular exercise can be attained without further burden of anxiolytics, antidepressants, or mood stabilizers. Given the positive effects of controlled exercise to improve metabolic responses, provide neuroprotection, increase quality of life, and reduce psychopathological symptoms, an individualized exercise prescription in the context of a monitored exercise program is an optimal and cost-effective method to manage the cardio-metabolic side effects of medication and should be widely available to those with severe mental illness.

\section{Compliance with Ethics Guidelines}

Conflict of Interest Ric M. Procyshyn is a paid consultant for and is on the speaker's bureau for AstraZeneca, Bristol-Myers Squibb, Janssen, Otsuka, Pfizer, and Sunovion.

Alasdair Barr has received consulting fees or sat on paid advisory boards for: Roche, Eli Lilly and Bristol-Myers Squibb. Donna Lang declares that she has no conflicts of interest.

Human and Animal Rights and Informed Consent This article does not contain any studies with human or animal subjects performed by any of the authors.

Open Access This article is distributed under the terms of the Creative Commons Attribution License which permits any use, distribution, and reproduction in any medium, provided the original author(s) and the source are credited.

\section{References}

Papers of particular interest, published recently, have been highlighted as:

- Of importance

•• Of major importance

1. Hofer A, Rettenbacher MA, Widschwendter CG, Kemmler G, Hummer M, Fleischhacker WW. Correlates of subjective and functional outcomes in outpatient clinic attendees with schizophrenia and schizoaffective disorder. Eur Arch Psychiat \& Clin Neurosci. 2006;256:246-55.

2. Hennekens CH, Hennekens AR, Hollar B, Casey DE. Schizophrenia and increased risk of cardiovascular disease. Amer Heart J. 2005;150:1115-21.

3. Kilbourne AM, Brar JS, Drayer RA, Xu X, Post EP. Cardiovascular disease and metabolic risk factors in male patients with schizophrenia, schizoaffective disorder, and bipolar disorder. Psychosomatics. 2007:48:412-7.

4. Association. CP: therapeutic choices. Ottawa, CPA. 2011

5. Bensenor IM, Brunoni AR, Pilan LA, Goulart AC, Busatto GF, Lotufo PA, et al. Cardiovascular disease in severe mental illness. Gen Hosp Psychiat. 2012;34:268-75.

6. Verma SK, Subramaniam M, Liew A, Poon LY. Metabolic risk factors in drug-naive patients with first-episode psychosis. J Clin Psychiat. 2009;70:997-1000.

7. Hasnain M, Victor W, Vieweg VR, Mezuk B. Mortality in schizophrenia. Neuropsychopharm. 2011;25:846-7.

8. Newcomer JW. Antipsychotic medications: metabolic and cardiovascular risk. J Clin Psychiat. 2007;68 Suppl 4:8-13.

9. Fagiolini A, Goracci A. The effects of undertreated chronic medical illnesses in patients with severe mental disorders. J Clin. 2009;70 Suppl 3:22-9.

10. Goeree R, Farahati F, Burke N, Blackhouse G, O'Reilly D, Pyne J, et al. The economic burden of schizophrenia in Canada in 2004. Curr Med Res Opin. 2005;21:2017-28.

11. Procyshyn RM, Honer WG, Wu TK, Ko RW, McIsaac SA, Young $\mathrm{AH}$, et al. Persistent antipsychotic polypharmacy and excessive dosing in the community psychiatric treatment setting: a review of medication profiles in 435 Canadian outpatients. J Clin Psychiat. 2004;71:566-73.

12. Kopala LC. Spontaneous and drug-induced movement disorders in schizophrenia. Acta Psychiatr Scand Suppl. 1996;389:12-7.

13. Meunch J, Hamer AM. Adverse effects of antipsychotic medications. Am Fam Phys. 2010;81:617-22.

14. Boyda HN, Tse L, Procyshyn RM, Honer WG, Barr AM. Preclinical models of antipsychotic drug-induced metabolic side-effects. Trends Pharmacol Sci. 2010;31:484-97.

15. Leung JY, Barr AM, Procyshyn RM, Honer WG, Pang CC. Cardiovascular side effects of antipsychotic drugs: the role of the autonomic nervous system. Pharmacol Ther. 2012;135:113-22.

16. Nyguyen D, Brakoulias V, Boyce P. An evaluation of monitoring practices in patients on second generation antipsychotics. Australas Psychiat. 2009;17:295-9.

17. Consensus development conference on antipsychotic drugs and obesity and diabetes. Diabetes Care. 2004;27:596-601.

18. Allison DB, Mentore JL, Heo M, Chandler LP, Cappelleri JC, Infante MC, et al. Antipsychotic-induced weight gain: a comprehensive research synthesis. Am J Psychiatry. 1999;156:1686-96.

19. Taylor DM, McAskill R. Atypical antipsychotics and weight gaina systematic review. Acta Psychiatr Scand. 2000;101:416-32.

20. Tandon R, Halbreich U. The second-generation 'atypical' antipsychotics: similar improved efficacy but different neuroendocrine side effects. Psychoneuroendocrinology. 2003;28 Suppl 1:1-7.

21. Ghaeli P, Dufresne RL. Elevated serum triglycerides on clozapine resolve with risperidone. Pharmacotherapy. 1995;15:382-3.

22. Henderson DC, Cagliero E, Gray C, Nasrallah RA, Hayden DL, Schoenfeld DA, et al. Clozapine, diabetes mellitus, weight gain, and lipid abnormalities: a five-year naturalistic study. Am J Psychiatry. 2000;157:975-81.

23. Lund BC, Perry PJ, Brooks JM, Arndt S. Clozapine use in patients with schizophrenia and the risk of diabetes, hyperlipidemia, and hypertension: a claims-based approach. Arch Gen Psychiatry. 2001;58:1172-6.

24. de Leon J, Susce MT, Johnson M, Hardin M, Pointer L, Ruano G, et al. A clinical study of the association of antipsychotics with hyperlipidemia. Schizophr Res. 2007;92:95-102.

25. AHA: Metabolic syndrome: new guidance for prevention and treatment. AHA \& NHLBI Scientific Statement, 2005.

26. McEvoy JP, Meyer JM, Goff DC, Nasrallah HA, Davis SM, Sullivan $\mathrm{L}$, et al. Prevalence of the metabolic syndrome in patients with schizophrenia: baseline results from the Clinical Antipsychotic Trials of Intervention Effectiveness (CATIE) schizophrenia trial and 
comparison with national estimates from NHANES III. Schizophr Res. 2005;80:19-32.

27. Newcomer JW, Haupt DW, Fucetola R, Melson AK, Schweiger JA, Cooper BP, et al. Abnormalities in glucose regulation during antipsychotic treatment of schizophrenia. Arch Gen Psychiatry. 2002;59:337-45.

28. Henderson DC, Cagliero E, Copeland PM, Borba CP, Evins E, Hayden D, et al. Glucose metabolism in patients with schizophrenia treated with atypical antipsychotic agents: a frequently sampled intravenous glucose tolerance test and minimal model analysis. Arch Gen Psychiatry. 2005;62:19-28.

29. Newcomer JW. Second-generation (atypical) antipsychotics and metabolic effects: a comprehensive literature review. CNS Drugs. 2005;19 Suppl 1:1-93.

30. Sacher J, Mossaheb N, Spindelegger C, Klein N, Geiss-Granadia $\mathrm{T}$, Sauermann R, et al. Effects of olanzapine and ziprasidone on glucose tolerance in healthy volunteers. Neuropsychopharmacology. 2008;33:1633-41.

31. Mitchell AJ, Delaffon V, Vancampfort D, Correll CU, De Hert M. Guideline concordant monitoring of metabolic risk in people treated wth antipsychotic medication: systematic review and metaanalysis of screening practices. Psychol Med. 2012;42:125-47.

32. Raedler TJ. Cardiovascular aspects of antipsychotics. Curr Opin Psychiatry. 2010;23:574-81.

33. De Hert M, Vancampfort D, Correll CU, Mercken V, Peuskens J, Sweers $\mathrm{K}$, et al. Guidelines for screening and monitoring of cardiometabolic risk in schizophrenia: systematic evaluation. Brit J Psychiatry. 2011;199:99-105.

34. Phillips AA, Warburton DE, Flynn SW, Fredikson D, Lang DJ. Physiological evidence of increased cardiovascular disease risk in schizophrenia-spectrum disorders: a pilot study using aortic pulse wave velocity and arterial compliance. Submitted. 2013.

35. Beebe LH, Smith KD, Roman MW, Burk RC, McIntyre K, Dessieux OL, et al. A pilot study describing phyiscal activity in persons with schizophrenia spectrum disorders (SSDS) after an exercise program. Issues Ment Health Nurs. 2013;34:214-9.

36. Knochel C, Oertel-Knochel V, O'Dwyer L, Prvulovic D, Alves G, Kollman B, et al. Cognitive and behavioural effects of physical exercise in psychiatric patients. Prog Neurobiol. 2012;92:46-68.

37. Blair SN, Brodney S. Effects of physical inactivity and obesity on morbidity and mortality: evidence and research issues. Med Sci Sports Exerc. 1999;31 Suppl 1:S646-57.
38. • Bernard P, Ninot G. Benefits of exercise for people with schizophrenia: a systematic review. Encephale. 2012;38:280-7. This recent review clearly tabulated our current evidence for the feasibility and/ or efficacy of exercise interventions for both psychiatric and physical remediation.

39. Alsuwaidan MT, Kucyi A, Law CWY, McIntyre RS. Exercise and bipolar disorder: a review of neurobiological mediators. Neuro Molec Med. 2009;11:328-36.

40. Babyak M, Blumenthal JA, Herman S, Khatri P, Doraiswamy M, Moore K, et al. Exercise treatment for major depression: maintenance of therapeutic benefit at 10 months. Psychsom Med. 2000;62:6338.

41. Beebe LH, Tian L, Morris N, Goodwin A, Allen SS, Kuldau J. Effects of exercise on mental and physical health parameters of persons with schizophrenia. Issues Ment Health Nurs. 2005;26:661-76.

42. Carter T, Callaghan P, Khalil E, Morres I. The effectiveness of a preferred intensity exercise programme on the mental health outcomes of young people with depression: a sequential mixed methods evaluation. BMC Publ Health. 2012;12:187.

43. - Lang DJ, Gigas K, Humphries BG, Honer WG, Smith GN, Thornton AE, et al. Effects of exercise on symptom severity and hippocampal volumes in treatment-resistant chronic schizophrenia: preliminary results. Proceedings of the International Congress of Schizophrenia Research, Orlando, Florida, Schizophrenia Bulletin. 2013;S340. Our new recent data demonstrate the feasibility and effectiveness of a managed exercise program in the most severely mentally ill patients to remediate mood deficits and improve physical fitness in the presence of high-dose atypical antipsychotic treatment.

44. Otte A, Hassler J, Brogowski J, Bowen JC, Mayhew JL. Relationship between body mass index and predicted $\%$ fat in college men and women. Mo J Health Physical Edn Rec Dance. 2000;10:23-9.

45. Tavazzi L, Dubach P, Opasich C, Meyers J, Perk J, Meyer K, et al. Working group report: recommendations for exercise testing in chronic heart failure patients. Eur Heart J. 2001;22:37-45.

46. Strassnig MT, Newcomer JW, Harvey PD. Exercise improves physical capacity in obese patients with schizophrenia: pilot study. Schiz Res. 2012;141:284-5.

47. Scheewe TW, Takken T, Kahn RS, Cahn W, Backx FJ. Effects of exercise therapy on cardiorespiratory fitness in patients with schizophrenia. Med Sci Sports Exerc. 2012;44:1834-42. 